\title{
A Research Review on Advanced Approches in Abrasive Jet Machining
}

\author{
S.Rajendra Prasad ${ }^{1}$,Dr.K.Ravindranath ${ }^{2}$ Dr. M.L.S.Devakumar ${ }^{3}$ \\ ${ }^{I}$ (Research Scholar, Mechanical Engineering, Jntua, Anathapur) \\ ${ }^{2}$ (Professor \& Dean, Mechanical Engineering, Svcet, Chittoor) \\ ${ }^{3}$ (Professor \& Vice Principal, Mechanical Engineering, Jntua, Anathapur)
}

\begin{abstract}
In the advanced areas of space, missile and nuclear technologies, there arise a need for machining components to maintain exact sharp edges, high accuracy, and precisely sized components. In the present era of modern machining, these requirements can be achieved with the help of advanced machining process like Abrasive Jet Machining (AJM).This paper presents an extensive review of the current state of research and development on abrasive jet machining process.
\end{abstract}

Keywords: Abrasive Jet Machine, Experimental, Mathematical, optimization approach.

\section{Abrasive Jet Machine (AJM):}

\section{Introduction}

Abrasives are very expensive but abrasive jet process requires low capital cost and operational cost because the investment on equipment is very low in comparison with other nontraditional mmachining processes giving tighter tolerances. As the carrier gas serves as a coolant, the cutting action is cool and hence better surface finish can be obtained. Now days it is widely used in manufacturing of electronic devices, LCD's, tribo-elements, MEMS, and semiconductors. There exists an increasing demand to develop micromachining technologies for these difficult-to-machine materials due to their properties of extreme hardness, brittleness, corrosion resistance and low melting temperatures. Abrasive jet machining (AJM) is a nontraditional machine process which operates without producing shocks and heat. In this machining process, the high - velocity stream of abrasives is generated by converting the pressure energy of carrier gas or air to its Kinetic energy and hence the high - velocity jet results. A nozzle directs abrasives in a controlled manner onto the work material. AJM is applied for many applications like cutting, cleaning, polishing, deburring, etching, drilling and finishing the operation. The nozzle is the most critical part in the abrasive air-jet equipment. The process is used chiefly to cut intricate shapes in hard and brittle materials which are sensitive to heat and have a tendency to chip easily. The process can be easily controlled by varying the parameters such as Velocity, Flow rate, Pressure, Standoff distance, Grit size, and nozzle angle. Response variables like surface finish, Material Removal Rate(MRR), kerfs width is producing a cylindrical hole.

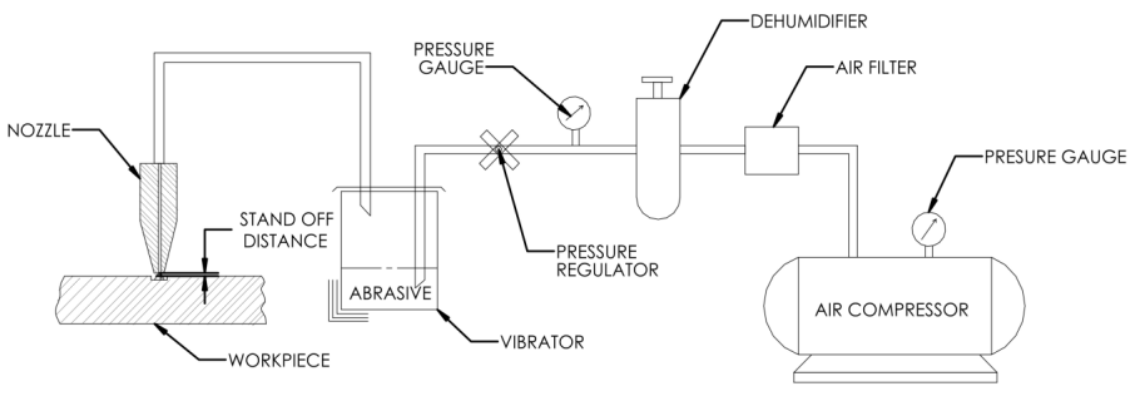

Fig1-Ajm Machine Model

\section{Back ground}

This novel technology was first demonstrated by Franz to cut laminated paper tubes in 1968 and was first introduced as a commercial system in 1983. In the 1980s garnet abrasive was added to the water stream and the abrasive jet was born. In the early 1990s, water jet pioneer Dr. John Olsen began to explore the concept of abrasive jet cutting as a practical alternative for traditional machine shops. His ultimate goal was to develop a system that could eliminate the noise, dust, and expertise demanded by abrasive jets at that time. In the last two 
decades, an extensive deal of research and development in AJM has been carried out. Based on the literature review of AJM Process the published works on this can be classified based on the performance measure considered into three different categories, namely experimental approach, Optimization approach and Analytical approach.

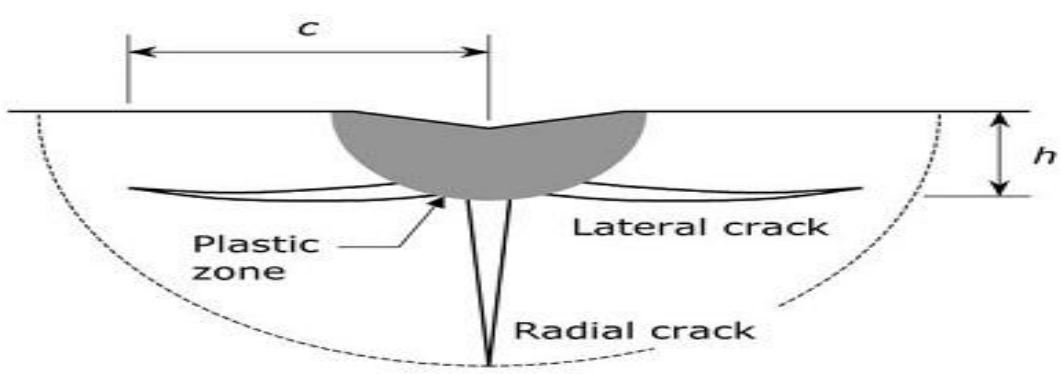

Fig2-Crack system when subjected to the indentation with a sharp indenter

\section{Literature Review}

In the review of AJM the contributions influencing the abrasive jet machining (AJM) process parameters on material removal rate, Surface integrity, and kerfs are discussed. The parameters like SOD, Carrier gas, Air Pressure, Type of Abrasive, Size, and Mixing Ratio etc.are focused.

\section{Experimental Approach}

V. C. Venkatesh [1] Performed Parametric Studies on Abrasive Jet Machining and demonstrated the effect of feed rate, pressure, abrasive grit size, Spray angle, nozzle tip to work distance, and metal removal rate and reported that wear is severe in the exit nozzle, considerable in the mixing chamber, and negligible in the inlet nozzle.

P.K.Ray \& Dr. Alepaul [2] reported a study on the results of machining under various conditions, compared the values and defined SOD as the main factor for material removal rate and plotted the graphs. In precision work, a higher pressure and a lower stand-off distance may be adopted to attain a, higher accuracy and penetration rate.

Parle et al. considered various parameters and found that when nozzle diameter is varied with machining of glass, the outcome under SEM analysis showed that U-type groove finishing is not that clear when compared to the machining square type grooves [7].

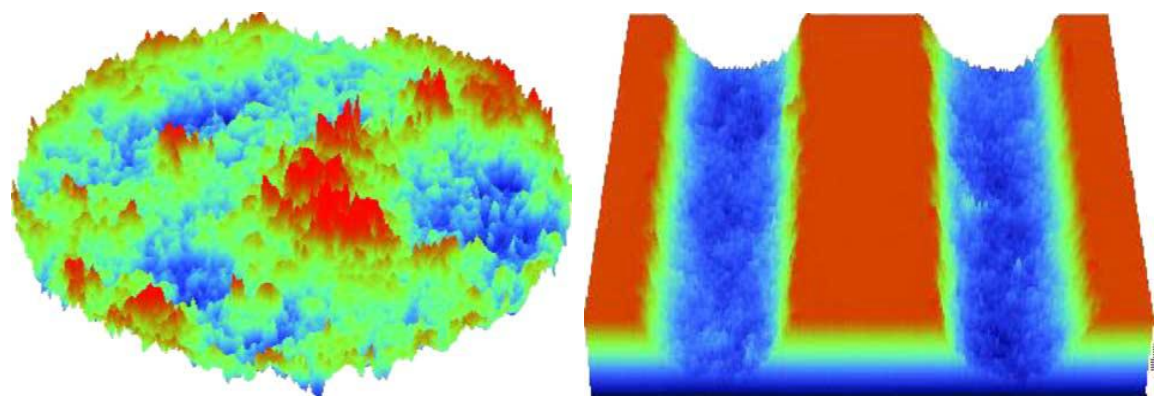

Fig3-Sem images of U Type \& Square type grooves

Massimiliano Barletta et al. [9] presented a paper identifying the material response of SS316L circular tubes by conducting FB-AJM basic level experiment. He concluded that decreasing abrasive sizes would improve the surface finish of the work piece and the tube descends from original $\mathrm{Ra} 1.5 \mathrm{~mm}$ to $\mathrm{Ra} 0.015 \mathrm{~mm}$. The authors of Hwan Lee et al. [12] newly developed a mask technology introducing SU-8 mask and watershed 11110 masks. When these two were applied on $\mathrm{Si}$ wafer material, the result showed that watershed mask performed better than SU-8 mask.

Yan-Cherng LIN et al explained the combination of AJM and EDM as Hybrid machining. A study was conducted and developed a novel hybrid process combining material removal mechanisms on AJM and EDM, then a series of experiments were conducted to determine the effects of the machining parameters and characteristics for machining of SKD 61 steel. The hybrid process of AJM and EDM can enhance the material 
removal. The abrasive grain generated mechanical plowing not only increased the MRR but also generated fine surface integrities. [16].

D.V. Srikanth \& M. Sreenivasa Rao Conducted experiments and analyzed the influence of process parameters on MRR and Kerfs width in abrasive jet Machining of Ceramic tile. The results of experiments was analyzed, It is observed that by increasing nozzle diameter the MRR increases, similarly decrease in Stand-off distance will reduce the divergence of the hole produced. [18]

Yan \& Zhu conducted an experiment considering the parameters velocity, jet angle and Ra on K9 glass. The photographic values of Ra were observed in 3D microscope. When the machining gap is maintained minimum then the surface finish of glass is improved [21].

Fritz Klocke et al. [25] Reviewed machinability of aerospace alloys and composites with recent developments involving novel/innovative grinding processes. He concentrated on perspectives relating to grinding in aerospace sector via, general work piece surface integrity standards/ requirements, fluid delivery systems, wheel preparation options and machine tool design/configurations.

R. H.M. Jafar et al. In their novel approach to reduce the dust in machining zone using water, glycerin and polymer solution in glass cutting to check response variables Ra, MRR. The results revealed that using glycerin solution reduce airborne dust by about $61 \%$, compared with long chain polymer $42 \%$ and pure water $36 \%$. When blasting through glycerin, better channel depth, width, erosion rate when compared to water. It also decreases roughness and increases channel centre line waviness. [26].

The researcher A. Nouhia et al focused on the parameters particle size, SOD with the newly developed shadow mask technology. There was an experimental comparison between shadow mask technology and clamped mask technology, The channels machined using the shadow mask were found to be approximately $10 \%$ wider than those machined using clamped masks up to an aspect ratio of approximately 0.5.with increasing nozzle standoff distance and mask thickness, decreasing mask standoff distance particle size leading to narrowest features with the least amount of frosting. [27].

Kumar Abhishek and Somashekhar S [30] made an attempt to overcome the conical shapes produced during machining operation on glass materials using the parameters feed rate, material removal, cylindricity, nozzle angle, in AJM. Entrance diameter of the hole reduces approximately by $29 \%$ whereas the taper angle is reduced by approximately $58 \%$, which results in the improved cylindricity, when tested under SEM analysis.
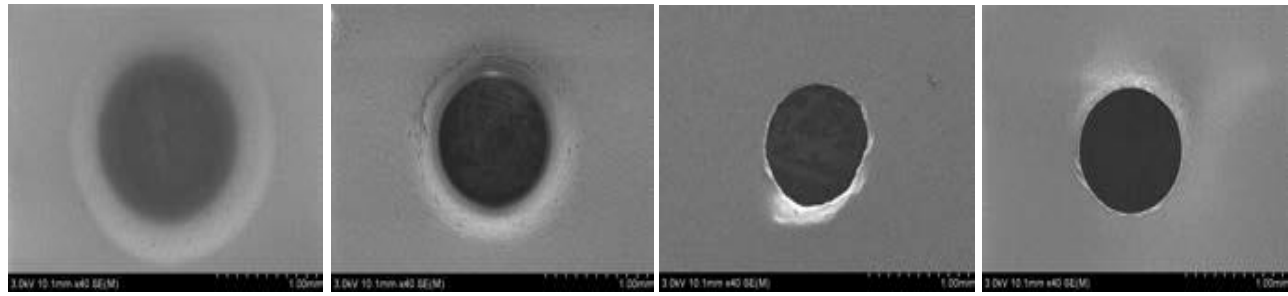

Fig4-SEM images of top and bottom diameter of the machined holes

\section{Optimization Approach}

R.Balasubramaniam et al. demonstrated the advantage of AJD over manual deburring SOD high edge generated parameter and compare the values using TAGUCHI \& ANOVA. [4].

Lei Zhanga Conducted experiments and analyzed the influence of process parameters on pressure, SOD and speed. The continuous flow of gas with abrasives from the nozzle could blow away any abrasive from a hole. This was analyzed under multiple regressions. Further experiments are conducted to confirm the validity of the developed statistical model by comparing the model predictions with the experimental results. [8].

Massimiliano Barletta conducted experiments with Inconel 718 material in FB-AJM considering various parameters, and the results obtained were analyzed using TAGUCHI analysis and concluded that the process produces fine surface finishes with minimal effect on the residual stress in the target surface. [10].

Srikanth \& Rao Conducted experiments on FRP composites and analyzed the influence of process parameters on MRR and Kerfs width. The results obtained from experiments were analyzed using Taguchi and ANOVA. The Optimal levels of Performance Found at Large is Better MRR was identified as air pressure (7 $\mathrm{kg} / \mathrm{cm} 2) \mathrm{SOD}(9 \mathrm{~mm})$ nozzle diameter $(2 \mathrm{~mm})$. The optimal level of performance found at smaller is Better Kerf was identified as air pressure $(7 \mathrm{~kg} / \mathrm{cm} 2)$ SOD $(7 \mathrm{~mm})$ Nozzle diameter $(3 \mathrm{~mm})$. [19].

Huaizhong $\mathrm{Li}$ an et al presented a paper considering pressure and velocity, using DEM-CFD analysis, Simulations of the particle flow characteristics using a range of particle shape factors are compared against experimental data with different inlet pressures and nozzle diameters It is found that the predicted results agree well with the experimental data with particle shape factors of 0.6 and 0.8 , which correspond to "edged" and "rounded" abrasive particles. [20]. 
In the paper presented by Baranitharan \& Zeelan Baslu two types of nozzles are combined for AJM process, elliptical and circular nozzle. The flow characteristics in these nozzles are analyzed using CFD analysis. The result shows that elliptical nozzle has an improvement up to $5 \%$ of mass flow rate compared to circular nozzle, without affecting the surface properties. [22].

R. Vijayaraghavan et al. in their paper stated, by conducting a machining process, by considering the parameters, abrasive particles and the key parameter machining time. In general the power loss and wastage losses are high to reduce that the parameters are analyzed by MARS-GP model with a trial and error method, this could avoid the loss of power and wastage . [24].

Srikanth \& Rao analyzed process parameters on MRR of CFRP composites using "Response surface methodology-RSM"order to obtain better results and optimal values. Obtained values after analysis are compared with ANOVA $S=0.00786696, \mathrm{R}-\mathrm{sq}=95.47 \%, \mathrm{R}-\mathrm{sq}(\mathrm{adj})=93.45 \%$ and $\mathrm{R}$-sq $(\mathrm{pred})=91.07 \%$ [28] .

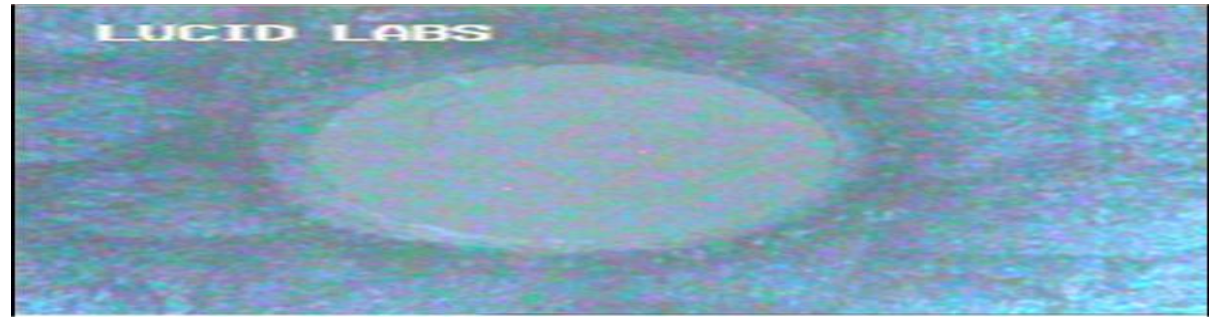

Fig5-Microstructure of CFRP after drilling

\section{Mathematical Approach}

Jeong-Du Kim et al. proposed a mathematical model using the parameters by considering the MRR and surface roughness is also increased by using of abrasive particles. [3].

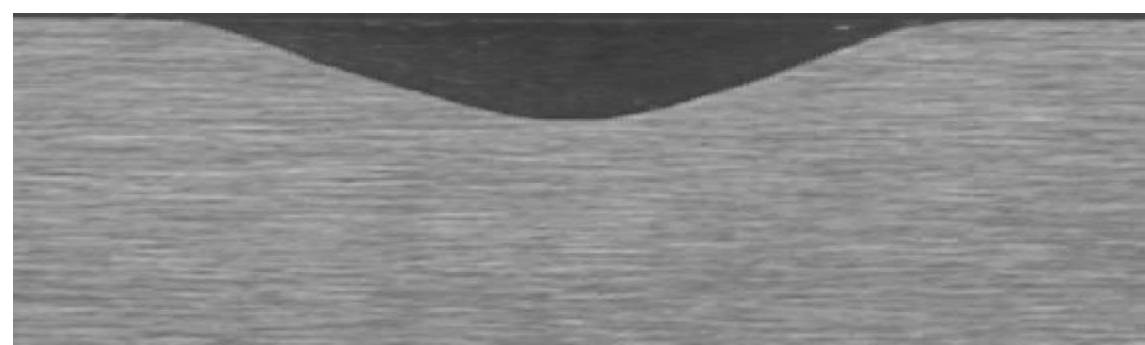

Fig6-Abrasive Jet Machined profile

R.Balasubramaniam et al. developed an equation for analyzing the parameters with in particle size increases the MRR increase, and also SOD increases as the edge radius increases. [5].

R Vijay Kumar in his thesis on machining of glass with different parameters, derived mathematical formulas with which when the SOD is maintained at $20 \mathrm{~mm}, 30 \mathrm{~mm}$ then the variations of MRR could be noticed. [6].

H Getu et al. Here, the author has chosen nozzle angle as the important parameter and prepared semiempirical model by fabricating new composite material called polymethylmethacrylate, and obtained results erosion rate on the impact angle was simulated very well using the semi-empirical model, showing that an increase in the erosion rate at $25^{\circ}$ angle of incidence. The temperature of the PMMA during abrasive jet micromachining of channels increase relative to the initial value was approximately $8 \circ \mathrm{C}$. It is recommended that if the erosion caused be ductile rather than brittle materials. [11].

In this paper EI Domiaty et al. has performed machining operation on glass material and developed a mathematical model and analyzed MRR. The experimental and theoretical results obtained for material removal rates are close to each other within an error of not more than 20 percent which can be accepted for a mathematical model based on an erosion model. [13].

The paper presented by Alizamoridi et al. says that the machining operation was performed on quartz crystal and developed a mathematical model, analyzed kerfs. It has been found that the predicted models provide good results for the range of conditions tested in this study. [14].

Y M Wan et al. in this paper presented that an analysis using AJM on soda lime glass and borosilicate glass was conducted, and a mathematical approach was simulated, resulting in an reasonable agreement between experimental values and predicted values. [15]. 
Haj Mohammad Jafari et al Investigated post-blasting particle size, velocity, dose, and angle of attack on the resulting reduction in roughness of reference channels in a borosilicate glass were demonstrated. Using post-blasting, the roughness of a reference channel can be decreased up to $60 \%$ of its initial value. Comparison with centerline roughness measurements for channels in borosilicate glass shows that the model can predict the transient roughness reduction in post-blasting particle dose with an error of $7 \%$ on average. [17].

The paper presented by N. S. Pawara et al, exploring the different parameters, nozzles, abrasive particles and simulated a cubic polynomial model to obtain MRR values experimentally. When the MRR is within limit the percentage of error is very less. MRR is directly proportional to pressure, when ever pressure is increased the MRR will slightly i.e. surface found to be $0.0289 \mathrm{gm} / \mathrm{sec}$ and at the pressure $5 \mathrm{~kg} / \mathrm{cm} 2 \mathrm{increase}$. Thus the result using the linear model for a polynomial reached $95 \%$ of the predicted. [23].

Here the author Zhen-Guo HE et al. Developed a numerical model to simulate by using the carrier gas SC-CO2 with considering the parameters pressure and velocity. The SC-CO2 gives a much higher particle impact velocity along with unique fluid properties, enables it to be better when compared with water jet. It also exhibits satisfactory carrying capacity, high two-phase velocity compared with abrasive water jet. [29].

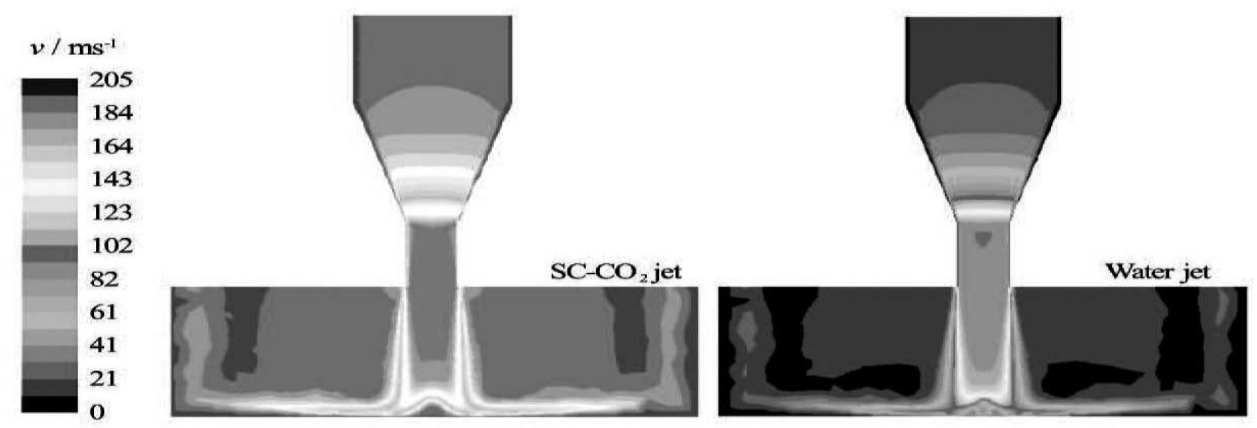

Fig7-Distributions of fluid velocities of abrasive SC-CO2 jet and abrasive water jet

\section{Conclusions}

An extensive review of the research and development in the AJM has been conducted in this paper. It was shown that AJM process is receiving more and more attention in the machining areas, particularly for the processing of difficult-to-cut materials. Its unique advantages over other conventional and un-conventional methods make it a new choice in the machining industry.

It is concluded that wide experimental investigations are required to fully understand the relationship between important AJM parameters, namely Air pressure, nozzle size and shape, abrasive mass flow rates and process output in greater detail for aluminum, brass, cast iron, ceramics, copper, composites, granite, mild steel, stainless steel and titanium as the right choice of process parameters is very important for good cutting performance.

There is much scope of research in the AJM which can be performed by changing the nozzle design, pressure, angle, SOD, etc. and Comparing the effect of various parameters on MRR on different metals like super alloys, composites, glass, and ceramics, by improving the cylindricity of the hole. And also Integration of AJM with CNC, model comparison, etc.are to be held in further stages. The mathematical model can be developed by replacing the conical shape hole with cylindrical hole.

\section{References}

[1]. V.C. Venkatesh "Parametric Studies on Abrasive Jet Machining" Annals of the CIRP vol33 No 1. Page 109. (1984)

[2]. P.K.Ray, Dr. Alepaul-“Some studies on AJM” IE, Vol68 part RE2. (1987)

[3]. Jeong-Du Kim *, Youn-Hee Kang, Young-Han Bae, Su-Won Lee "Development of a magnetic abrasive jet machining system for precision internal polishing of circular tubes" Elsevier Journal of Materials Processing Technology 71 ( 1997) 384-393

[4]. R.Balasubramaniam, J Krishnan, N Ramakrishna "Investigation of AJM for deburring” Elsevier 2002

[5]. R.Balasubramaniam, J Krishnan, N Ramakrishna "A study on the shape of the surface generated by AJM" Elsevier 2002.

[6]. R.Vijay Kumar “An investigation into the mechanics of AJM (Thesis)” NIT, Calicut 2002.

[7]. Parle, Cho, Lee, co "Micro-grooving of glass using micro AJM" Elsevier, Journal of material process technology 2004.

[8]. Lei Zhang,*, Tsunemoto Kuriyagawab, Yuya Yasutomi, Ji Zhao "Investigation into micro abrasive intermittent jet machining” Elsevier International Journal of Machine Tools \& Manufacture 45 (2005) 873-879. 
[9]. Massimiliano Barletta*, Vincenzo Tagliaferri "Development of an abrasive jet machining system assisted by two fluidized beds for internal polishing of circular tubes" Elsevier International Journal of Machine Tools \& Manufacture 46 (2006) 271-283

[10]. M.Barletta, ceccarelli.S,S.Guarino,V.Tagliaferri "Fluidized Bed Assisted Abrasive Jet Machining „FB-AJM Precision Internal Finishing of Inconel 718 Components" ASME,Vol. 129 / 1045-2007

[11]. H Getu, A Ghobeily, J K spelt, M Papini “AJMM of polymethylmethacrylate” Elsevier, wear 263-2007.

[12]. In Hwan Lee, Seung-Pyo Lee, Tae Jo Ko, Hyun Wook Kang and Dong-Woo Cho "Development of Rapid Mask Fabrication Technology for Micro-Abrasive Machining" IEEE 2008.

[13]. A EI Domiaty, H M Ajad EL - Haf-Ez, M A Shaker "Drilling of glass sheets by AJM" IEEE 2009.

[14]. Alizamoridi, Junwang, Yassear "A study of abrasive jet micro grooving of quartz crystal" Scientific journal key engage materials vol 443(2010)

[15]. Y M Wan, W S Fong, Q Y Leong "Deterministic process modeling of the micro air abrasive jet machinery of glass channel” Scientific journal sim tech technical reports (STR_v12_N2_03_MTG) vol 12 numbers 02-2011.

[16]. Yan-cherry line, Chu, SEI "Machining performance on the hybrid process of AJM and EDM" Elsevier, non-ferrous2012.

[17]. Haj Mohammad Jafari, M. Papini b,a,*, J.K. Spelta "Simulation of erosive smoothing in the abrasive jet micromachining of glass" Elsevier Journal of Materials Processing Technology-2013.

[18]. D.V. Srikanth, M. Sreenivasa Rao "Application of optimization methods on AJM of ceramics" IJIET vol.04 issue03-2014.

[19]. D.V. Srikanth, M. Sreenivasa Rao "Machining of FRP composites by AJM optimization using Taguchi method" IEEE-2014.

[20]. Huaizhong Li a, Ann Lee a, Jingming Fan a, Guan Heng Yeoh, JunWang“On DEM-CFD study of the dynamic characteristics of high-speed micro-abrasive air jet" Elsevier Powder Technology-2014.

[21]. Yan, Zhu "Study on processing characteristics of constrained abrasive jet polishing" IEEE-2014.

[22]. Baranitharan, Zeelan Baslu "Design, and analysis of elliptical nozzle in AJM process using CFD" Sci Technol arts Resh 4 (1) 171 - 179 (2015).

[23]. N. S. Pawar, R.R. Lakhe, R. L. Shrivastava "Validation of Experimental Work by Using Cubic Polynomial Models For Sea Sand as an Abrasive Material in Silicon Nozzle in AJM" Materials Today: Proceedings 2 ( 2015 ) 1927 1933

[24]. R. Vijayaraghavan a, A. Garg b, $\Uparrow$, V. Vijayaraghavan b, Liang Gao "Development of energy consumption model of abrasive machining process by a combined evolutionary computing approach" Elsevier Measurement-2015.

[25]. Fritz Klocke, Sein Leung Soo, Bernhard Karpuschewski, John A. Webster. "Abrasive machining of advanced aerospace alloys and composites" Elsevier CIRP Annals - Manufacturing Technology-2015.

[26]. R. H.M. Jafar, V. Hadavi, J.K. Spelt, M. Papini "Dust Reduction in Abrasive Jet Micro-machining Using Liquid Films" IEEE-2015.

[27]. A. Nouhi, M.R. Sookhak Laria,J.K. Spelt, M. Papini,* "Implementation of a shadow mask for direct writing in abrasive jet micro-machining" Elsevier Journal of Materials Processing Technology-2015.

[28]. D.V.Srikanth, Dr.M.Sreenivasa Rao "Application of RSM for the optimal response of Process parameters on machining of CFRP composites by using AJM" IEEE-2016.

[29]. Zhen-guo HE, Gen-sheng LI, Hai-zhu WANGZhong-hou SHEN "Numerical simulation of the abrasive supercritical carbon dioxide jet: The flow field and the influencing factors" Elsevier journal of hydro dynamics-2016.

[30]. Kumar Abhishek and Somashekhar S Hiremath "Improvement of Geometrical Accuracy of Micro Holes Machined Through Micro AJM” Elsevier Procedia CIRP 46 (2016) 47 - 50 